\title{
Inverse spectral problem for Sturm-Liouville operator with discontinuous coefficient and cubic polynomials of spectral parameter in boundary condition
}

Aynur Çöl*

\section{"Correspondence: \\ aynurcol@sinop.edu.tr \\ Department of Mathematics \\ Education, Sinop University, Sinop,}

57100, Turkey

\begin{abstract}
In this paper, the inverse scattering problem for the Sturm-Liouville operator with discontinuous coefficient and cubic polynomials of the spectral parameter in the boundary condition is considered. The scattering data of the problem is defined, and its properties are investigated. The modified Marchenko main equation is obtained and it is shown that the potential is uniquely recovered by the scattering data.
\end{abstract}

MSC: 34A55; 34B40; 34B07; 34L25; 81U40

Keywords: inverse problem; scattering data; Sturm-Liouville operator; spectral parameter; discontinuous coefficient

\section{Introduction}

Consider the boundary value problem generated by the differential equation

$$
-y^{\prime \prime}+q(x) y=\lambda^{2} \rho(x) y \quad(0<x<\infty)
$$

with the boundary condition

$$
\left(\alpha_{0}+i \alpha_{1} \lambda-\alpha_{2} \lambda^{2}-i \alpha_{3} \lambda^{3}\right) y^{\prime}(0)-\left(\beta_{0}+i \beta_{1} \lambda-\beta_{2} \lambda^{2}-i \beta_{3} \lambda^{3}\right) y(0)=0
$$

where $\lambda$ is a spectral parameter, $q(x)$ is real valued function with the condition

$$
\int_{0}^{\infty}(1+x)|q(x)| d x<\infty
$$

and $\rho(x)$ is a piecewise constant function in the form

$$
\rho(x)= \begin{cases}\alpha^{2}, & 0 \leq x<a \\ 1, & a \leq x<\infty\end{cases}
$$

$1 \neq \alpha>0$. Here $p_{j}(\lambda)(j=1,2)$ is a polynomial

$$
p_{1}(\lambda) \equiv \alpha_{0}+i \alpha_{1} \lambda-\alpha_{2} \lambda^{2}-i \alpha_{3} \lambda^{3}, \quad p_{2}(\lambda) \equiv \beta_{0}+i \beta_{1} \lambda-\beta_{2} \lambda^{2}-i \beta_{3} \lambda^{3}
$$
tional License (http://creativecommons.org/licenses/by/4.0/), which permits unrestricted use, distribution, and reproduction in any medium, provided you give appropriate credit to the original author(s) and the source, provide a link to the Creative Commons license, and indicate if changes were made. 
with the relations

$$
\alpha_{i+1} \beta_{i}-\alpha_{i} \beta_{i+1}>0, \quad \alpha_{i+2} \beta_{i}-\alpha_{i} \beta_{i+2}<0, \quad \alpha_{i+3} \beta_{i}-\alpha_{i} \beta_{i+3}=0
$$

for $\alpha_{i}, \beta_{i} \in \mathbb{R}(i=\overline{0,3})$.

Inverse spectral problems of spectral analysis often appear in mathematics, mechanics, physics and other branches of natural sciences. The direct scattering problem consists of the determination the collection $\left\{S(\lambda),\left\{\lambda_{j}\right\}_{j=1}^{N},\left\{m_{j}\right\}_{j=1}^{N}\right\}$ when $q(x)$ is known. The inverse scattering problem deals with the construction of $q(x)$ in terms of the scattering data. In this paper in the case of discontinuous coefficient (4), we consider the inverse scattering problem for Sturm-Liouville operator with cubic polynomials of spectral parameter in boundary condition (2).

Discontinuous inverse problems appear in electronics for constructing parameters of heterogeneous electronic lines with desirable technical characteristics and in geophysical models for oscillations of the earth (see [1] and the references therein). In the case that $\rho(x) \equiv 1$ and the boundary condition does not contain a spectral parameter, the inverse scattering problem for (1) was solved by Marchenko [2, 3] and Levitan [4, 5]. The inverse scattering problem of the Sturm-Liouville operator with discontinuous coefficient was studied in [6-8]. The problem was examined in [9] by using a new integral representation of the Jost solution of (1).

An important case in spectral theory is that containing the spectral parameter in equations and boundary conditions. Sturm-Liouville problems with spectral parameterdependent boundary conditions arise in studies of heat conduction problems and vibrating string problems. Fulton and Pruess showed a kind of heat conduction problems in [10]. Problems with the dependence on spectral parameter can be found in [10-17]. The inverse scattering problem for (1) with a linear spectral parameter in the boundary condition was solved in [18].

The main result of this paper is that the potential $q(x)$ can be uniquely recovered from the given scattering data. The Marchenko method is applied to solving the boundary value problem when the boundary conditions depend on spectral parameter as nonlinear.

If $q(x)=0$, the result is obtained that the function

$$
f_{0}(x, \lambda)=\frac{1}{2}\left(1+\frac{1}{\sqrt{\rho(x)}}\right) e^{i \lambda \mu^{+}(x)}+\frac{1}{2}\left(1-\frac{1}{\sqrt{\rho(x)}}\right) e^{i \lambda \mu^{-}(x)}
$$

is a solution of $(1)$, where $\mu^{ \pm}(x)= \pm x \sqrt{\rho(x)}+a(1 \mp \sqrt{\rho(x)})$.

As proven in [9], if the condition (3) is satisfied, (1) has a unique solution $f(x, \lambda)$, which satisfies the asymptotic behavior

$$
\lim _{x \rightarrow+\infty} e^{-i \lambda x} f(x, \lambda)=1
$$

for $\operatorname{Im} \lambda \geq 0$ and can be expressed by

$$
f(x, \lambda)=f_{0}(x, \lambda)+\int_{\mu^{+}(x)}^{\infty} K(x, t) e^{i \lambda t} d t
$$


which is called the Jost solution. For the kernel function $K(x, t)$, the inequality

$$
\int_{\mu^{+}(x)}^{\infty}|K(x, t)| d t \leq c\left(\exp \left(\int_{x}^{\infty} t|q(t)| d t\right)\right), \quad 0<c=\text { const }
$$

is satisfied. Also, if $q(x)$ is differentiable, the kernel $K(x, t)$ is twice differentiable and satisfies both the equation

$$
\frac{\partial^{2} K(x, t)}{\partial x^{2}}-\rho(x) \frac{\partial^{2} K(x, t)}{\partial t^{2}}=q(x) K(x, t), \quad 0<x<\infty, t>\mu^{+}(x),
$$

and the conditions

$$
\begin{aligned}
& \frac{d K\left(x, \mu^{+}(x)\right)}{d x}=-\frac{1}{4 \sqrt{\rho(x)}}\left(1+\frac{1}{\sqrt{\rho(x)}}\right) q(x), \\
& \frac{d}{d x}\left\{K\left(x, \mu^{-}(x)+0\right)-K\left(x, \mu^{-}(x)-0\right)\right\}=\frac{1}{4 \sqrt{\rho(x)}}\left(1-\frac{1}{\sqrt{\rho(x)}}\right) q(x) .
\end{aligned}
$$

Denote by $\varphi(\lambda, x)$ the solution of (1) satisfying the conditions

$$
\varphi(0, \lambda)=p_{1}(\lambda), \quad \varphi^{\prime}(0, \lambda)=p_{2}(\lambda) .
$$

This solution satisfies the condition (2). The function

$$
S(\lambda)=\frac{p_{1}(\lambda) \overline{f^{\prime}(0, \lambda)}-p_{2}(\lambda) \overline{f(0, \lambda)}}{p_{1}(\lambda) f^{\prime}(0, \lambda)-p_{2}(\lambda) f(0, \lambda)}
$$

is the scattering function of the boundary value problem (1)-(3). It is a meromorphic function in the upper half plane $\operatorname{Im} \lambda>0$ with the poles at the zeros of the function $p_{1}(\lambda) f^{\prime}(0, \lambda)-p_{2}(\lambda) f(0, \lambda)$. These poles are simple and lie on the imaginary axis. The norming numbers are defined as

$$
\begin{aligned}
m_{k}^{-2} \equiv & \int_{0}^{\infty} f^{2}\left(x, i \mu_{k}\right) \rho(x) d x+\frac{f^{2}\left(0, i \mu_{k}\right)}{p_{1}^{2}\left(i \mu_{k}\right)}\left[\frac{1}{2} \sum_{m=0}^{2}\left(\alpha_{1+m} \beta_{m}-\alpha_{m} \beta_{1+m}\right) \mu_{k}^{2 m-1}\right. \\
& \left.+\sum_{m=0}^{1}\left(\alpha_{m} \beta_{2+m}-\alpha_{2+m} \beta_{m}\right) \mu_{k}^{2 m}\right]
\end{aligned}
$$

where $\lambda_{k}=i \mu_{k}, \mu_{k}>0$. We show that the kernel $K(x, y)$ of the solution (6) satisfies the integral equation which is called the main equation,

$$
\begin{aligned}
& K(x, y)+F(x, y) \\
& \quad+\int_{\mu^{+}(x)}^{\infty} K(x, t) F_{0}(t+y) d t+\frac{1-\sqrt{\rho(x)}}{1+\sqrt{\rho(x)}} K(x, 2 a-y)=0, \quad y>\mu^{+}(x),
\end{aligned}
$$

where

$$
F(x, y)=\frac{1}{2}\left(1+\frac{1}{\sqrt{\rho(x)}}\right) F_{0}\left(y+\mu^{+}(x)\right)+\frac{1}{2}\left(1-\frac{1}{\sqrt{\rho(x)}}\right) F_{0}\left(y+\mu^{-}(x)\right),
$$




$$
\begin{aligned}
& F_{0}(x)=\frac{1}{2 \pi} \int_{-\infty}^{\infty}\left[S_{\infty}(\lambda)-S(\lambda)\right] e^{i \lambda x} d \lambda+\sum_{k=1}^{N} m_{k}^{2} e^{-\mu_{k} x}, \quad \lambda_{k}=i \mu_{k}, \\
& S_{\infty}(\lambda)= \begin{cases}e^{-2 i \lambda a} \frac{\tau e^{-2 i \lambda a \alpha}-1}{\tau-e^{-2 i \lambda a \alpha}}, & \alpha_{n} \neq 0, \\
e^{-2 i \lambda a \frac{\alpha \alpha_{2}\left(-1+\tau e^{-2 i \lambda a \alpha}\right)-\beta_{3}\left(1+\tau e^{-2 i \lambda a \alpha}\right)}{\alpha \alpha_{2}\left(e^{-2 i \lambda a \alpha}-\tau\right)-\beta_{3}\left(e^{-2 i \lambda a \alpha}+\tau\right)},} & \alpha_{n}=0\end{cases}
\end{aligned}
$$

and $\tau=\frac{\alpha-1}{\alpha+1}$.

Obviously, from the given scattering data $\left\{S(\lambda),\left\{\lambda_{j}\right\}_{j=1}^{N},\left\{m_{j}\right\}_{j=1}^{N}\right\}$, the function $F(x, y)$ is found by (12) which is then introduced in (11). If (11) has a unique solution $K(x, y)$, then the function $q(x)$ can be found from (7) and (8). The solvability of the integral equation (11) is examined and the algorithm of recovering the potential $q(x)$ is given.

This paper is organized as follows. In Section 2, the scattering data to the boundary value problem (1)-(3) is found by using a new integral representation for the solution of (1), and its properties are investigated. In Section 3, the main equation for the boundary value problem (1)-(3) is derived. Finally, the solvability of the main equation is proved and the unique recovery of the potential from the solution of the main equation is shown in Section 4.

Let $y(x, \lambda)$ and $z(x, \lambda)$ be solutions of (1). The expression

$$
W[y(x, \lambda), z(x, \lambda)]=y^{\prime} z-y z^{\prime}
$$

is called the Wronskian of the functions $y(x, \lambda)$ and $z(x, \lambda)$. It is clear that for all real $\lambda \neq 0$, $f(x, \lambda)$ and $\overline{f(x, \lambda)}$ constitute fundamental solutions of (1). The Wronksian of these functions does not depend on $x$ and equals $2 i \lambda$.

\section{Scattering data}

Lemma 1 For all real $\lambda \neq 0$, the following identity is valid:

$$
2 i \lambda \frac{\varphi(x, \lambda)}{E(\lambda)}=\overline{f(x, \lambda)}-S(\lambda) f(x, \lambda)
$$

and $S(\lambda)$ possesses the following properties:

$$
S(\lambda)=\overline{S(-\lambda)}, \quad|S(\lambda)|<1
$$

Proof Since $f(x, \lambda)$ and $\overline{f(x, \lambda)}$ constitute the fundamental solution system of (1) for real $\lambda \neq 0$, we have

$$
\varphi(x, \lambda)=c_{1}(\lambda) f(x, \lambda)+c_{2}(\lambda) \overline{f(x, \lambda)}
$$

where $c_{1}(\lambda)$ and $c_{2}(\lambda)$ are functions which we have to find. Taking account of the following equalities:

$$
\begin{aligned}
& c_{1}(\lambda) f(0, \lambda)+c_{2}(\lambda) \overline{f(0, \lambda)}=p_{1}(\lambda), \\
& c_{1}(\lambda) f^{\prime}(0, \lambda)+c_{2}(\lambda) \overline{f^{\prime}(0, \lambda)}=p_{2}(\lambda),
\end{aligned}
$$


$c_{1}(\lambda)$ and $c_{2}(\lambda)$ are found and substituted in (14). Thus, we obtain

$$
\varphi(x, \lambda)=-\frac{p_{1}(\lambda) \overline{f^{\prime}(0, \lambda)}-p_{2}(\lambda) \overline{f(0, \lambda)}}{2 i \lambda} f(x, \lambda)+\frac{p_{1}(\lambda) f^{\prime}(0, \lambda)-p_{2}(\lambda) f(0, \lambda)}{2 i \lambda} \overline{f(x, \lambda)} .
$$

Let

$$
E(\lambda) \equiv p_{1}(\lambda) f^{\prime}(0, \lambda)-p_{2}(\lambda) f(0, \lambda)
$$

Now, it is necessary to show $E(\lambda) \neq 0$ for all real $\lambda \neq 0$. Assume the contrary, then there exists $\lambda_{0} \in \mathbb{R}, \lambda_{0} \neq 0$, such that

$$
p_{1}\left(\lambda_{0}\right) f^{\prime}\left(0, \lambda_{0}\right)=p_{2}\left(\lambda_{0}\right) f\left(0, \lambda_{0}\right)
$$

Also

$$
W\left[f\left(0, \lambda_{0}\right), \overline{f\left(0, \lambda_{0}\right)}\right]=2 i \lambda_{0}
$$

is satisfied. By using these relations, we obtain

$$
\frac{\left|f\left(0, \lambda_{0}\right)\right|^{2}}{\left|p_{1}\left(\lambda_{0}\right)\right|^{2}}\left[\alpha_{1} \beta_{0}-\alpha_{0} \beta_{1}+\left(\alpha_{2} \beta_{1}-\alpha_{1} \beta_{2}\right)\left|\lambda_{0}\right|^{2}+\left(\alpha_{3} \beta_{2}-\alpha_{2} \beta_{3}\right)\left|\lambda_{0}\right|^{4}\right]=-1
$$

and this is a contradiction since the left-hand side is positive. Thus, by dividing the equality (15) by $\frac{1}{2 i \lambda} E(\lambda)$, we obtain (13) where $S(\lambda)$ is defined with (9). Since $\overline{E(\lambda)}=E(-\lambda)$ and the numerator has the same property, it is clear that

$$
\overline{S(\lambda)}=S(-\lambda)
$$

Also $\left[p_{1}(\lambda) \overline{p_{2}(\lambda)}-p_{2}(\lambda) \overline{p_{1}(\lambda)}\right]\left[f^{\prime}(0, \lambda) \overline{f(0, \lambda)}-\overline{f^{\prime}(0, \lambda)} f(0, \lambda)\right]<0$ holds for all real $\lambda \neq 0$, and so the identity

$$
|S(\lambda)|^{2}<1
$$

is satisfied. Thus, the lemma is proved.

Lemma 2 The function $E(\lambda)$ has only a finite number of zeros on the half plane $(\operatorname{Im} \lambda>0)$. All the zeros are simple and lie on the imaginary axis.

Proof By the proof of Lemma 1 we have $E(\lambda) \neq 0$ for all real $\lambda \neq 0$, the point $\lambda=0$ is the possible zero of $E(\lambda)$. Since $E(\lambda)$ is analytic in the upper plane $\operatorname{Im} \lambda>0$ and $f(0, \lambda)$ is bounded as $|\lambda| \rightarrow \infty$, it follows that the set of zeros of $E(\lambda)$ is bounded and forms at most countable set having as zero the only possible limit point.

Now we show that zeros of $E(\lambda)$ lie on the imaginary axis. Assume that $\lambda_{1}$ and $\lambda_{2}$ are zeros of $E(\lambda)$. Then they satisfy (1):

$$
\begin{aligned}
& -f^{\prime \prime}\left(x, \lambda_{1}\right)+q(x) f\left(x, \lambda_{1}\right)=\lambda_{1}^{2} \rho(x) f\left(x, \lambda_{1}\right), \\
& -\overline{f^{\prime \prime}\left(x, \lambda_{2}\right)}+q(x) \overline{f\left(x, \lambda_{2}\right)}=\overline{\lambda_{2}^{2}} \rho(x) \overline{f\left(x, \lambda_{2}\right)} .
\end{aligned}
$$


We multiply (16) by $\overline{f\left(x, \lambda_{2}\right)}$ and (17) by $f\left(x, \lambda_{1}\right)$, subtract the latter from the former, and finally integrate this relation according to $x$ from 0 to $\infty$. As a result,

$$
\left(\lambda_{1}^{2}-\overline{\lambda_{2}^{2}}\right) \int_{0}^{\infty} f\left(x, \lambda_{1}\right) \overline{f\left(x, \lambda_{2}\right)} \rho(x) d x-\left.W\left[f\left(x, \lambda_{1}\right), \overline{f\left(x, \lambda_{2}\right)}\right]\right|_{x=0}=0 .
$$

On the other hand, since $\lambda_{j}(j=1,2)$ is a zero to $E(\lambda)$ we have

$$
\left.W\left[f\left(x, \lambda_{1}\right), \overline{f\left(x, \lambda_{2}\right)}\right]\right|_{x=0}=\left(\frac{p_{2}\left(\lambda_{1}\right)}{p_{1}\left(\lambda_{1}\right)}-\frac{\overline{p_{2}\left(\lambda_{2}\right)}}{\overline{p_{1}\left(\lambda_{2}\right)}}\right) f\left(0, \lambda_{1}\right) \overline{f\left(0, \lambda_{2}\right)} .
$$

If we take $\lambda_{1}=\lambda_{2}=\mu$ and substitute in (18), the result is obtained that

$$
\begin{aligned}
& (\mu+\bar{\mu})\left[\frac{|f(0, \mu)|^{2}}{\left|p_{1}(\mu)\right|^{2}}\left[\sum_{k=0}^{2}\left(\alpha_{1+k} \beta_{k}-\alpha_{k} \beta_{1+k}\right)|\mu|^{2 k}+2 \operatorname{Im} \mu \sum_{k=0}^{1}\left(\alpha_{k} \beta_{2+k}-\alpha_{2+k} \beta_{k}\right)|\mu|^{2 k}\right]\right. \\
& \left.\quad+2 \operatorname{Im} \mu \int_{0}^{\infty}|f(x, \mu)|^{2} \rho(x) d x\right]=0 .
\end{aligned}
$$

Since we have the condition (5), the expression in the parentheses is positive, and it implies that $\mu+\bar{\mu}=0$, i.e., $\mu$ is pure imaginary.

Let us prove that there are only finitely many zeros. Let $\delta$ denote the infimum of the distances between two neighboring zeros of $E(\lambda)$, and show $\delta>0$. Let us assume the contrary and let $\left\{i \lambda_{k}\right\}$ and $\left\{i \hat{\lambda}_{k}\right\}$ be two sequences of zeros of the function $E(\lambda)$ such that

$$
\lim _{k \rightarrow \infty}\left(\hat{\lambda}_{k}-\lambda_{k}\right)=0, \quad 0<\lambda_{k}<\hat{\lambda}_{k}, \quad \max _{k} \hat{\lambda}_{k}<M
$$

For $A$ large enough, the inequality

$$
f\left(x, i \lambda_{k}\right)>\frac{1}{2} e^{-\lambda_{k} x}
$$

holds uniformly with respect to $x \in[A, \infty)$ and $\lambda \in[0, \infty)$. Thus, we obtain

$$
\int_{A}^{\infty} f\left(i \hat{\lambda}_{k}, x\right), \overline{f\left(i \lambda_{k}, x\right)} \rho(x) d x>\frac{1}{4} \frac{e^{-A\left(\hat{\lambda}_{k}+\lambda_{k}\right)}}{\left(\hat{\lambda}_{k}+\lambda_{k}\right)}>\frac{e^{-2 A M}}{8 M} .
$$

On the other hand, the equality (18) yields

$$
\begin{aligned}
0= & \frac{f\left(0, i \hat{\lambda}_{k}\right) \overline{f\left(0, i \lambda_{k}\right)}}{\overline{p_{1}\left(i \lambda_{k}\right)} p_{1}\left(i \hat{\lambda}_{k}\right)}\left[\sum_{m=0}^{2}\left(\alpha_{1+m} \beta_{m}-\alpha_{m} \beta_{1+m}\right)\left(\hat{\lambda}_{k} \lambda_{k}\right)^{m}\right. \\
& \left.+\sum_{m=0}^{1}\left(\alpha_{m} \beta_{2+m}-\alpha_{2+m} \beta_{m}\right)\left(\hat{\lambda}_{k} \lambda_{k}\right)^{m}\left(\hat{\lambda}_{k}+\lambda_{k}\right)\right] \\
& +\left(\hat{\lambda}_{k}+\lambda_{k}\right) \int_{0}^{\infty} f\left(x, i \hat{\lambda}_{k}\right) \overline{f\left(x, i \lambda_{k}\right)} \rho(x) d x \\
= & \left(\hat{\lambda}_{k}+\lambda_{k}\right) \int_{0}^{A} f\left(x, i \hat{\lambda}_{k}\right)\left[\overline{f\left(x, i \lambda_{k}\right)}-\overline{f\left(x, \hat{i} \hat{\lambda}_{k}\right)}\right] \rho(x) d x
\end{aligned}
$$




$$
\begin{aligned}
& +\left(\hat{\lambda}_{k}+\lambda_{k}\right) \int_{0}^{A} f\left(x, i \hat{\lambda}_{k}\right) \overline{f\left(x, i \hat{\lambda}_{k}\right)} \rho(x) d x \\
& +\left(\hat{\lambda}_{k}+\lambda_{k}\right) \int_{A}^{\infty} f\left(x, i \hat{\lambda}_{k}\right) \overline{f\left(x, i \lambda_{k}\right)} \rho(x) d x \\
& +\frac{f\left(0, i \hat{\lambda}_{k}\right) \overline{f\left(0, i \lambda_{k}\right)}}{\overline{p_{1}\left(i \lambda_{k}\right)} p_{1}\left(i \hat{\lambda}_{k}\right)}\left[\sum_{m=0}^{2}\left(\alpha_{1+m} \beta_{m}-\alpha_{m} \beta_{1+m}\right)\left(\hat{\lambda}_{k} \lambda_{k}\right)^{m}\right. \\
& \left.+\sum_{m=0}^{1}\left(\alpha_{m} \beta_{2+m}-\alpha_{2+m} \beta_{m}\right)\left(\hat{\lambda}_{k} \lambda_{k}\right)^{m}\left(\hat{\lambda}_{k}+\lambda_{k}\right)\right]
\end{aligned}
$$

and letting $k \rightarrow \infty$, we get

$$
\lim _{k \rightarrow \infty} \int_{A}^{\infty} f\left(x, i \hat{\lambda}_{k}\right) \overline{f\left(x, i \lambda_{k}\right)} \rho(x) d x \leq 0 .
$$

Since

$$
\lim _{k \rightarrow \infty}\left[\overline{f\left(x, i \lambda_{k}\right)}-\overline{f\left(x, i \hat{\lambda}_{k}\right)}\right]=0
$$

uniformly with respect to $x \in[0, A]$. Comparing (19) and (20) we reach a contradiction. We conclude that $\delta>0$ and so the function $E(\lambda)$ has only a finite number of zeros.

Now, let us show that all zeros of the function $E(\lambda)$ are simple. By the derivation of the identity

$$
-f^{\prime \prime}(x, \lambda)+q(x) f(x, \lambda)=\lambda^{2} \rho(x) f(x, \lambda)
$$

with respect to $\lambda$, we get

$$
-\dot{f}^{\prime \prime}(x, \lambda)+q(x) \dot{f}(x, \lambda)=\lambda^{2} \rho(x) \dot{f}(x, \lambda)+2 \lambda \rho(x) f(x, \lambda),
$$

here $\dot{f}$ denotes differentiation with respect to $\lambda$. Multiplying (21) by $\dot{f}(x, \lambda)$ and (22) by $f(x, \lambda)$ and subtracting the second from the first and integrating this relation with respect to $x$ over $(0, \infty)$, the result is obtained that

$$
2 \lambda \int_{0}^{\infty} f^{2}(x, \lambda) \rho(x) d x+\left.W[f(x, \lambda), \dot{f}(x, \lambda)]\right|_{x=0}=0
$$

Let $\lambda$ be a zero of the function $E(\lambda)$. By using the expression for the function $E(\lambda)$, it is found that

$$
\begin{aligned}
\frac{\dot{E}(\lambda) f(0, \lambda)}{p_{1}(\lambda)}= & 2 \lambda \int_{0}^{\infty} f^{2}(x, \lambda) \rho(x) d x+i \frac{f^{2}(0, \lambda)}{p_{1}^{2}(\lambda)}\left[\sum_{m=0}^{2}\left(\alpha_{1+m} \beta_{m}-\alpha_{m} \beta_{1+m}\right)(i \lambda)^{2 m}\right. \\
& \left.+\sum_{m=0}^{1}\left(\alpha_{2+m} \beta_{m}-\alpha_{m} \beta_{2+m}\right)(i \lambda)^{2 m+1}\right] .
\end{aligned}
$$

Substituting $\lambda_{k}=i \mu_{k}, \mu_{k}>0$, in (23) and multiplying by $-i$ the result is obtained that the right side of the equality is positive. Thus $E\left(\dot{i \mu_{k}}\right) \neq 0$, i.e. the zeros of $E(\lambda)$ are simple. The lemma is proved. 
The numbers

$$
\begin{aligned}
m_{k}^{-2} \equiv & \int_{0}^{\infty} f^{2}\left(x, i \mu_{k}\right) \rho(x) d x+\frac{f^{2}\left(0, i \mu_{k}\right)}{p_{1}^{2}\left(i \mu_{k}\right)}\left[\frac{1}{2} \sum_{m=0}^{2}\left(\alpha_{1+m} \beta_{m}-\alpha_{m} \beta_{1+m}\right) \mu_{k}^{2 m-1}\right. \\
& \left.+\sum_{m=0}^{1}\left(\alpha_{m} \beta_{2+m}-\alpha_{2+m} \beta_{m}\right) \mu_{k}^{2 m}\right]
\end{aligned}
$$

are called norming numbers.

The collection $\left\{S(\lambda),\left\{\lambda_{k}\right\}_{k=1}^{N},\left\{m_{k}\right\}_{k=1}^{N}\right\}$ is called the scattering data for the boundary value problem (1)-(3).

Using (6) and substituting the related expressions into $S(\lambda)$, the following result is obtained:

$$
S(\lambda)=e^{-2 i \lambda a} \frac{\tau e^{-2 i \lambda a \alpha}-1}{e^{-2 i \lambda a \alpha}-\tau}+O\left(\frac{1}{\lambda}\right)
$$

if $\alpha_{3} \neq 0$ as $|\lambda| \rightarrow \infty$, and

$$
S(\lambda)=e^{-2 i \lambda a} \frac{\alpha \alpha_{2}\left(-1+\tau e^{-2 i \lambda a \alpha}\right)-\beta_{3}\left(1+\tau e^{-2 i \lambda a \alpha}\right)}{\alpha \alpha_{2}\left(e^{-2 i \lambda a \alpha}-\tau\right)-\beta_{3}\left(e^{-2 i \lambda a \alpha}+\tau\right)}+O\left(\frac{1}{\lambda}\right)
$$

if $\alpha_{3}=0$ as $|\lambda| \rightarrow \infty$.

Let

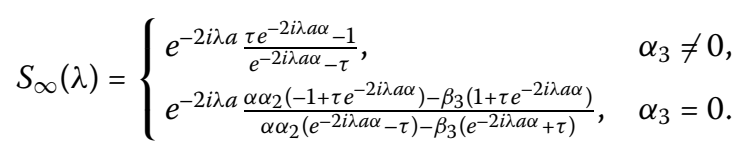

Hence $S_{\infty}(\lambda)-S(\lambda) \in L_{2}(-\infty, \infty)$ and so the function

$$
F_{0 S}(x)=\frac{1}{2 \pi} \int_{-\infty}^{\infty}\left(S_{\infty}(\lambda)-S(\lambda)\right) e^{i \lambda x} d \lambda
$$

also belongs to the space $L_{2}(-\infty, \infty)$.

\section{The main equation}

The inverse scattering problem consists in recovering the coefficient $q(x)$ from the scattering data. It is clear that in order to determine $q(x)$ it is sufficient to know the kernel $K(x, t)$ of the solution (6). To derive the integral equation for $K(x, t)$, we use the equality (13), which was obtained in Lemma 1. Rewriting the identity (13) we get the following form:

$$
\begin{aligned}
& \frac{2 i \lambda \varphi(\lambda, x)}{E(\lambda)}-\overline{f_{0}(x, \lambda)}+S_{\infty}(\lambda) f_{0}(x, \lambda) \\
& =\int_{\mu^{+}(x)}^{\infty}\left[S_{\infty}(\lambda)-S(\lambda)\right] K(x, t) e^{i \lambda t} d t+\left[S_{\infty}(\lambda)-S(\lambda)\right] f_{0}(x, \lambda) \\
& \quad+\int_{\mu^{+}(x)}^{\infty} K(x, t) e^{-i \lambda t} d t-\int_{\mu^{+}(x)}^{\infty} S_{\infty}(\lambda) K(x, t) e^{i \lambda t} d t .
\end{aligned}
$$


Multiplying both sides of this equality by $\frac{1}{2 \pi} e^{i \lambda y}$ and integrating it to $\lambda$ from $-\infty$ to $\infty$, it is found that

$$
\begin{aligned}
& \frac{1}{2 \pi} \int_{-\infty}^{\infty}\left[\frac{2 i \lambda \varphi(\lambda, x)}{E(\lambda)}-\overline{f_{0}(x, \lambda)}+S_{\infty}(\lambda) f_{0}(x, \lambda)\right] e^{i \lambda y} d \lambda \\
& =\frac{1}{2 \pi} \int_{-\infty}^{\infty}\left[S_{\infty}(\lambda)-S(\lambda)\right] f_{0}(x, \lambda) e^{i \lambda y} d \lambda \\
& \quad+\frac{1}{2 \pi} \int_{-\infty}^{\infty} \int_{\mu^{+}(x)}^{\infty} K(x, t) e^{-i \lambda(t-y)} d t d \lambda-\frac{1}{2 \pi} \int_{-\infty}^{\infty} \int_{\mu^{+}(x)}^{\infty} S_{\infty}(\lambda) K(x, t) e^{i \lambda(t+y)} d t d \lambda \\
& \quad+\frac{1}{2 \pi} \int_{-\infty}^{\infty} \int_{\mu^{+}(x)}^{\infty}\left[S_{\infty}(\lambda)-S(\lambda)\right] K(x, t) e^{i \lambda(t+y)} d t d \lambda .
\end{aligned}
$$

To compute the third term on the right, we need to find new expression for $S_{\infty}(\lambda)$. After some calculations, in the case $\alpha_{3}=0$ we have

$$
\begin{aligned}
S_{\infty}(\lambda) & =e^{-2 i \lambda a} \frac{\left(\alpha \alpha_{2}+\beta_{3}\right)\left(\tau^{2}-1\right) e^{2 i \lambda a \alpha}}{\left(\alpha \alpha_{2}-\beta_{3}\right)-\left(\alpha \alpha_{2}+\beta_{3}\right) \tau e^{2 i \lambda a \alpha}}+\tau e^{-2 i \lambda a} \\
& =e^{-2 i \lambda a(1-\alpha)}\left(\tau^{2}-1\right) \sum_{k=0}^{\infty}\left(\frac{\alpha \alpha_{2}+\beta_{3}}{\alpha \alpha_{2}-\beta_{3}}\right)^{k+1} \tau^{k} e^{2 i \lambda a \alpha k}+\tau e^{-2 i \lambda a}
\end{aligned}
$$

and then

$$
\begin{aligned}
& \frac{1}{2 \pi} \int_{-\infty}^{\infty} S_{\infty}(\lambda) e^{i \lambda(t+y)} d \lambda \\
& \quad=\left(\tau^{2}-1\right) \sum_{k=0}^{\infty}\left(\frac{\alpha \alpha_{2}+\beta_{3}}{\alpha \alpha_{2}-\beta_{3}}\right)^{k+1} \tau^{k} \delta(t+y-2 a(1-\alpha)+2 a \alpha k)+\tau \delta(t+y-2 a) .
\end{aligned}
$$

Hence, the right-hand side of (24) equals

$$
\begin{aligned}
& K(x, y)+F_{S}(x, y)+\int_{\mu^{+}(x)}^{\infty} K(x, t) F_{0 S}(t+y) d t-\tau K(x, 2 a-y) \\
& -\left(\tau^{2}-1\right) \sum_{k=0}^{\infty}\left(\frac{\alpha \alpha_{2}+\beta_{3}}{\alpha \alpha_{2}-\beta_{3}}\right)^{k+1} \tau^{k} K(x, 2 a(1-\alpha)-2 a \alpha k-y),
\end{aligned}
$$

where

$$
\begin{aligned}
& F_{0 S}(x)=\frac{1}{2 \pi} \int_{-\infty}^{\infty}\left[S_{\infty}(\lambda)-S(\lambda)\right] e^{i \lambda x} d \lambda, \\
& F_{S}(x, y)=\frac{1}{2}\left(1+\frac{1}{\sqrt{\rho(x)}}\right) F_{0 S}\left(y+\mu^{+}(x)\right)+\frac{1}{2}\left(1-\frac{1}{\sqrt{\rho(x)}}\right) F_{0 S}\left(y+\mu^{-}(x)\right) .
\end{aligned}
$$

We note that $K(x, y)=0$ for $y<\mu^{+}(x)$. If $0<x<a$, then $\mu^{+}(x)=\alpha x-\alpha a+a$, and so we have for $k=0,1, \ldots$,

$$
2 a(1-\alpha)-2 a \alpha k-y<2 a(1-\alpha)-2 a \alpha k-\alpha x+\alpha a-a=a-\alpha a-2 a \alpha k-\alpha x \leq \mu^{+}(x) .
$$

Hence, this shows that the last term is equal to zero. If $x \geq a$, then $\mu^{+}(x)=x$, and the inequality holds for this case. 
Therefore, the right-hand side of (24) becomes

$$
K(x, y)+F_{S}(x, y)+\int_{\mu^{+}(x)}^{\infty} K(x, t) F_{0 S}(t+y) d t-\frac{1-\sqrt{\rho(x)}}{1+\sqrt{\rho(x)}} K(x, 2 a-y), \quad y>\mu^{+}(x) .
$$

In the case $\alpha_{3} \neq 0$,

$$
S_{\infty}(\lambda)=e^{-2 i \lambda a} \frac{\left(\tau^{2}-1\right) e^{2 i \lambda a \alpha}}{1-\tau e^{2 i \lambda a \alpha}}+\tau e^{-2 i \lambda a}=e^{-2 i \lambda a(1-\alpha)}\left(\tau^{2}-1\right) \sum_{k=0}^{\infty} \tau^{k} e^{2 i \lambda a \alpha k}+\tau e^{-2 i \lambda a}
$$

and we arrive the same result of the right of (24).

On the other side, using the residue theorem and Jordan's lemma we have

$$
\frac{1}{2 \pi} \int_{-\infty}^{\infty}\left[\frac{2 i \lambda \varphi(\lambda, x)}{E(\lambda)}-\overline{f_{0}(x, \lambda)}+S_{\infty}(\lambda) f_{0}(x, \lambda)\right] e^{i \lambda y} d \lambda=-\sum_{k=1}^{n} \frac{2 i \lambda_{k} \varphi\left(i \lambda_{k}, x\right)}{\dot{E}\left(i \lambda_{k}\right)} e^{-\lambda_{k} y}
$$

Taking (23) into account we can transform this expression to the form

$$
\begin{aligned}
& -\sum_{k=1}^{n} \frac{2 i \lambda_{k} \varphi\left(i \lambda_{k}, x\right)}{\dot{E}\left(i \lambda_{k}\right)} e^{-\lambda_{k} y} \\
& =-\sum_{k=1}^{n} \frac{2 i \lambda_{k} p_{1}\left(i \lambda_{k}\right)}{f\left(0, i \lambda_{k}\right) \dot{E}\left(i \lambda_{k}\right)} f\left(x, i \lambda_{k}\right) e^{-\lambda_{k} y}=-\sum_{k=1}^{n} m_{k}^{2} f\left(x, i \lambda_{k}\right) e^{-\lambda_{k} y} \\
& =-\sum_{k=1}^{n} m_{k}^{2}\left[f_{0}\left(x, i \lambda_{k}\right) e^{-\lambda_{k} y}+\int_{\mu^{+}(x)}^{\infty} K(x, t) e^{-\lambda_{k}(t+y)} d t\right]
\end{aligned}
$$

Substituting this value into the left side of (24), we obtain (11).

Thus we arrive at the following theorem.

Theorem 1 For every fixed $x \geq 0$, the kernel $K(x, t)$ to the special solution (6) satisfies the integral equation (11).

The integral equation (11) is called the main equation for the boundary value problem (1)-(3). The main equation does not have same form as the classical Marchenko equation and we call (11) the modified Marchenko equation.

\section{Solvability of the main equation}

We construct (11) only on the basis of the given scattering data. In this equation, we can take kernel $K(x, t)$ as unknown and regard it as a Fredholm-type equation for every fixed $x$. The main equation is rewritten in the form

$$
\begin{aligned}
& K\left(x, y+\mu^{+}(x)\right)+F\left(x, y+\mu^{+}(x)\right)+v K\left(x, 2 a-y-\mu^{+}(x)\right) \\
& \quad+\int_{0}^{\infty} K\left(x, t+\mu^{+}(x)\right) F_{0}\left(t+y+2 \mu^{+}(x)\right) d t=0, \quad y>0 .
\end{aligned}
$$

Theorem 2 For every fixed $x \geq 0$, the main equation (11) has a unique solution $K(x, \cdot) \in$ $L_{1}\left(\mu^{+}(x), \infty\right)$. 
Proof Let

$$
f(y)=K\left(x, y+\mu^{+}(x)\right)
$$

For the proof of the solvability of the given main equation, it is enough to show that the homogeneous equation

$$
f(y)+v K\left(x, 2 a-y-\mu^{+}(x)\right)+\int_{0}^{\infty} f(t) F_{0}\left(t+y+2 \mu^{+}(x)\right) d t=0
$$

has no nontrivial solution in the corresponding space.

If $f(y) \in L_{1}(0, \infty)$ is a solution of (26), then both $f(y) \in L_{\infty}(0, \infty)$ and $f(y) \in L_{2}(0, \infty)$. Hence $f(y) \in L_{1}(0, \infty) \cap L_{\infty}(0, \infty) \subset L_{2}(0, \infty)$ and it is sufficient to investigate (26) in $L_{2}(0, \infty)$. Equation (26) has the same properties as the fundamental equation of the problem in [18]. The proof of this fact is analog to Lemma 4.2 and Corollary 4.3 in [18].

Multiplying (26) by $\overline{f(y)}$ and integrating it from $-\infty$ to $\infty$ with respect to $y$, we obtain

$$
\begin{aligned}
& \frac{1}{2 \pi} \int_{\infty}^{\infty}|\widetilde{f(\lambda)}|^{2} d \lambda+\frac{1}{2 \pi} \int_{\infty}^{\infty} v e^{2 i \lambda \mu^{+}(x)-2 i \lambda a} \widetilde{f(-\lambda)} \widetilde{\widetilde{f(\lambda)}} d \lambda+\sum_{k=1}^{n} m_{k}^{2} e^{-2 \lambda_{k} \mu^{+}(x)}\left|\widetilde{f\left(-i \lambda_{k}\right)}\right|^{2} \\
& \quad+\frac{1}{2 \pi} \int_{\infty}^{\infty}\left(S_{\infty}(\lambda)-S(\lambda)\right) e^{2 i \lambda \mu^{+}(x)} \widetilde{f(-\lambda)} \widetilde{f(\lambda)} d \lambda=0
\end{aligned}
$$

and hence

$$
\begin{aligned}
\frac{1}{2 \pi} \int_{\infty}^{\infty}|\widetilde{f(\lambda)}|^{2} d \lambda & =\frac{1}{2 \pi} \int_{\infty}^{\infty} S(\lambda) e^{2 i \lambda \mu^{+}(x)} \widetilde{f(-\lambda)} \widetilde{f(\lambda)} d \lambda-\sum_{k=1}^{n} m_{k}^{2} e^{-2 \lambda_{k} \mu^{+}(x)}\left|\widetilde{f\left(-i \lambda_{k}\right)}\right|^{2} \\
& \leq \frac{1}{2 \pi} \int_{\infty}^{\infty}\left|S(\lambda) e^{2 i \lambda \mu^{+}(x)} \widetilde{f(-\lambda) \widetilde{f(\lambda)}}\right| d \lambda \leq\left.\frac{1}{2 \pi} \int_{\infty}^{\infty}|S(\lambda)| \widetilde{f(\lambda)}\right|^{2} d \lambda,
\end{aligned}
$$

i.e.,

$$
\int_{\infty}^{\infty}(1-|S(\lambda)|)|\widetilde{f(\lambda)}|^{2} d \lambda \leq 0
$$

Since $1-|S(\lambda)|>0$ for all $\lambda \neq 0$, this implies that $\widetilde{f(\lambda)} \equiv 0$. Therefore, the homogeneous equation (26) has only the null solution, and this proves the theorem.

Theorem 3 The scattering data of the boundary value problem (1)-(3) determines the potential $q(x)$ in (1) uniquely.

Proof Obviously, to form the main equation (11) it is sufficient to know the matrix function $F(x, y)$ and in its turn, to find $F(x, y)$ it is sufficient to know the scattering data $\left\{S(\lambda),\left\{\lambda_{j}\right\}_{j=1}^{N},\left\{m_{j}\right\}_{j=1}^{N}\right\}$. It is seen in Theorem 2 that the main equation (11), constructed only on the basis of the scattering data, has a unique solution $K(x, y)$. Then the function $q(x)$ in (1) can be uniquely found according to (7) and (8). Equation (1) is constructed by the given algorithm. The theorem is proved. 
Author's contributions

The author carried out the research of this paper, and she read and approved the final version of the manuscript.

\section{Acknowledgements}

This work is supported by The Scientific and Technological Research Council of Turkey (TÜBITAK)

Received: 9 December 2014 Accepted: 17 April 2015 Published online: 29 April 2015

\section{References}

1. Shieh, CT, Yurko, VA: Inverse nodal and inverse spectral problems for discontinuous boundary value problems. J. Math. Anal. Appl. 347, 266-272 (2008)

2. Marchenko, VA: Sturm-Liouville Operators and Applications. Birkhäuser, Basel (1986)

3. Marchenko, VA: On reconstruction of the potential energy from phases of the scattered waves. Dokl. Akad. Nauk SSSR 104, 695-698 (1955)

4. Levitan, BM: The inverse scattering problem of quantum theory. Mat. Zametki 17(4), 611-624 (1975)

5. Levitan, BM: Inverse Sturm-Liouville Problems. VSP, Zeist (1987). Translated from the Russian by O Efimov

6. Gasymov, MG: Direct and inverse problems of spectral analysis for a class of equations with discontinuous coefficients. In: Proceedings of the International Conference on Non-Classical Methods in Geophysics, Novosibirsk, pp. 37-44 (1977) (in Russian)

7. Darwish, AA: The inverse scattering problem for a singular boundary value problem. N.Z. J. Math. 22, 37-56 (1993)

8. Darwish, AA: On a direct and inverse scattering problem for a boundary value problem with discontinuous coefficient. N.Z. J. Math. 25, 1-14 (1996)

9. Guseinov, IM, Pashaev, RT: On an inverse problem for a second-order differential equation. Russ. Math. Surv. 57(3) 597-598 (2002)

10. Fulton, CT, Pruess, S: Numerical methods for a singular eigenvalue problem with eigenparameter in the boundary conditions. J. Math. Anal. Appl. 71, 431-462 (1979)

11. Walter, J: Regular eigenvalue problems with eigenvalue parameter in the boundary conditions. Math. Z. 133, 301-312 (1973)

12. Fulton, CT: Two-point boundary value problems with eigenvalue parameter contained in the boundary conditions. Proc. R. Soc. Edinb., Sect. A 77, 293-308 (1977)

13. Binding, PA, Browne, PJ, Watson, BA: Inverse spectral problems for Sturm-Liouville equations with eigenparameter dependent boundary conditions. J. Lond. Math. Soc. 62, 161-182 (2000)

14. Chugunova, MV: Inverse spectral problem for the Sturm-Liouville operator with eigenvalue parameter dependent boundary conditions. Oper. Theory, Adv. Appl. 123, 187-194 (2001)

15. Chernozhukova, A, Freiling, G: A uniqueness theorem for the boundary value problems with non-linear dependence on the spectral parameter in the boundary conditions. Inverse Probl. Sci. Eng. 17(6), 777-785 (2009)

16. Yurko, VA: An inverse problem for pencils of differential operators. Mat. Sb. 191(10), 137-160 (2000)

17. Mamedov, KR, Kosar, NP: Inverse scattering problem for Sturm-Liouville operator with nonlinear dependence on the spectral parameter in the boundary condition. Math. Methods Appl. Sci. 34, 231-241 (2011)

18. Mamedov, KR: On an inverse scattering problem for a discontinuous Sturm-Liouville equation with a spectral parameter in the boundary condition. Bound. Value Probl. 2010, 171967 (2010)

\section{Submit your manuscript to a SpringerOpen ${ }^{\odot}$ journal and benefit from:}

$\checkmark$ Convenient online submission

- Rigorous peer review

- Immediate publication on acceptance

- Open access: articles freely available online

- High visibility within the field

- Retaining the copyright to your article 